\title{
Selected topics on quadrature domains *
}

\author{
Björn Gustafsson ${ }^{a}$ \\ ${ }^{a}$ Department of Mathematics, Royal Institute of Technology, Stockholm S-100 44, \\ Sweden \\ Mihai Putinar ${ }^{b}$ \\ ${ }^{\mathrm{b}}$ Department of Mathematics, University of California, Santa Barbara, CA 93106
}

\begin{abstract}
This is a selection of facts, old and new, about quadrature domains. The text, written in the form of a survey, is addressed to non-experts and covers a variety of phenomena related to quadrature domains. Such as: the difference between quadrature domains for subharmonic, harmonic and respectively complex analytic functions, geometric properties of the boundary, instability in the reverse Hele-Shaw flow, dependence and non-uniqueness on the quadrature data, interpretation in terms of function theory on Riemann surfaces, a matrix model and a reconstruction algorithm. Plus some low degree/order examples where computations can be carried out in detail.
\end{abstract}

Key words: Quadrature domain, potential, free boundary, Markov moment problem, principal function

\section{Introduction}

Finite nodes, Gaussian type quadratures are traditionally constructed for polynomials of a fixed degree. The idea of studying planar domains which carry

^ The first author was partially supported by the Swedish research Council and by the Göran Gustafsson Foundation. The second author was partially supported by the NSF grant DMS 0350911

Email addresses: gbjorn@kth.se (Björn Gustafsson), mputinar@math.ucsb.edu (Mihai Putinar).

URLs: www.math.kth.se $\backslash^{\sim}$ gbjorn (Björn Gustafsson), www.math.ucsb.edu $\Upsilon^{\sim}$ mputinar (Mihai Putinar).

Preprint submitted to Elsevier Science 29 September 2006 
a finite node quadrature formula which is valid for all analytic (integrable) functions has independently appeared, for different motivations and almost simultaneously, in the works of Philip Davis [12], Dov Aharonov and Harold S. Shapiro [2], and Makoto Sakai [64], [65]. The mere elaboration of the concept and the discovery of the basic properties of quadrature domains for analytic, harmonic, or subharmonic functions have revealed exciting new territories, paved with an array of intriguing questions (some of them open still today) and with ramifications to quite a few topics of classical mathematics.

The golden age of quadrature domains has started about thirty years ago and was marked by the visionary ideas and works of H. S. Shapiro and his group. As basic references for their contributions we cite [16], [72] and the more recent volume [59]. Via S. Richardson's paper [60] connections to Hele-Shaw flow moving boundary problems (Laplacian growth) were revealed at an early stage, and the two subjects have then developed largely in parallel. For example, construction of quadrature domains using potential theoretic methods [65], [66] and the equivalent theory of weak (variational inequality) solutions for Hele-Shaw problems [13], [17] were obtained almost simultaneously, and essentially independent, around 1980.

The evolution of the field has not been short of surprising new turns. A major event was the discovery [54], [55] in the early 1990:s that quadrature domains are highly relevant within the theory of hyponormal operators. The connection is nontrivial and tools from operator theory, for example the determinantal function which now bears the name exponential transform, has virtually revolutionized the theory of quadrature domains.

Another big surprise was the appearance of Laplacian growth problems, both in deterministic and probabilistic forms, in a variety of branches of modern physics, like integrable systems, Toda lattice hierarchies, string theory, 2D quantum gravity, DLA, random matrices. Examples of relevant articles in this respect are [80], [47], [79], [42], [48], [45], [1], [43], [74], [33], [85]. See also [32]. Other papers in this volume will certainly witness of this development

We do not aim below at writing a self-contained mathematical introduction to quadrature domains, the recent survey [31] serving better for this scope. Rather, we offer the reader a quick glimpse at a selection of subjects related to quadrature domains, with emphasis on some phenomena and constructs which might be relevant to the group of participants to the meeting "Growing Interfaces, Los Alamos, 2006."

\section{Contents}

1 Introduction

2 Subharmonic quadrature domains and Moving boundaries 
$\begin{array}{lll}3 & \text { Algebraic domains } & 7\end{array}$

$4 \quad$ Signed measures, instability, uniqueness $\quad 8$

$5 \quad$ Markov's moment problem 12

$\begin{array}{lll}6 & \text { The trace formula } & 14\end{array}$

$\begin{array}{lll}7 & \text { A matrix model for quadrature domains } & 16\end{array}$

$\begin{array}{lll}8 & \text { The exponential transform } & 18\end{array}$

$\begin{array}{lll}9 & \text { A reconstruction algorithm } & 19\end{array}$

10 Examples 20

$\begin{array}{ll}10.1 \text { Reconstruction of a disk } & 20\end{array}$

10.2 Quadrature domains with a double node 21

10.3 Quadrature domains with two distinct nodes 22

10.4 Three points, non-simply connected quadrature domains and the non-uniqueness phenomenon 22

$\begin{array}{ll}\text { References } & 25\end{array}$

\section{Subharmonic quadrature domains and Moving boundaries}

If $\varphi$ is a subharmonic function (i.e., $\Delta \varphi \geq 0$ ) then the submeanvalue property

$$
\varphi(a) \leq \frac{1}{|B(a, r)|} \int_{B(a, r)} \varphi \mathrm{dA}
$$

holds for any disc $B(a, r) \subset \mathbb{C}$ contained in the domain of definition of $\varphi$. Above, and throughout this article $d A$ stands for Lebesgue dA. Thus, with $\Omega=B(a, r), c=|B(a, r)|=\pi r^{2}$ and $\mu=c \delta_{a}$ there holds

$$
\int \varphi \mathrm{d} \mu \leq \int_{\Omega} \varphi \mathrm{dA}
$$

for all subharmonic functions $\varphi$ in $\Omega$. This set of inequalities says that $\Omega$ is a quadrature domain for subharmonic functions with respect to $\mu$ [65], and it expresses that $\Omega=B(a, r)$ is a swept out version of the measure $\mu=c \delta_{a}$. If $c$ 
increases the corresponding expansion of $\Omega$ is a simple example of Hele-Shaw evolution, or Laplacian growth.

The above can be repeated with finitely many points, i.e., with $\mu$ of the form

$$
\mu=c_{1} \delta_{a_{1}}+\ldots+c_{n} \delta_{a_{n}}
$$

$a_{j} \in \mathbb{C}, c_{j}>0$ : there always exists a unique (up to nullsets) open set $\Omega \subset \mathbb{C}$ such that (1) holds for all $\varphi$ subharmonic and integrable in $\Omega$. One can think of it as the union $\bigcup_{j=1}^{n} B\left(a_{j}, r_{j}\right), r_{j}=\sqrt{c_{j} / \pi}$, with all multiple coverings smashed out to a singly covered set, $\Omega$. In particular, $\bigcup_{j=1}^{n} B\left(a_{j}, r_{j}\right) \subset \Omega$.

The above sweeping process, $\mu \mapsto \Omega$, or better $\mu \mapsto \chi_{\Omega} \cdot(\mathrm{dA})$, called partial balayage [65], [28], [21], applies to quite general measures $\mu \geq 0$ and can be defined in terms of a natural energy minimization: Given $\mu, \nu=\chi_{\Omega} \cdot(\mathrm{dA})$ will be the unique solution of

$$
\begin{aligned}
& \operatorname{Minimize}_{\nu}\|\mu-\nu\|_{e}^{2}: \\
& \nu \leq \mathrm{dA}, \int \mathrm{d} \nu=\int \mathrm{d} \mu .
\end{aligned}
$$

Here $\|\cdot\|_{e}$ is the energy norm:

$$
\begin{gathered}
\|\mu\|_{e}^{2}=(\mu, \mu)_{e}, \quad \text { with } \\
(\mu, \nu)_{e}=\frac{1}{2 \pi} \int \log \frac{1}{|z-\zeta|} d \mu(z) d \nu(\zeta) .
\end{gathered}
$$

If $\mu$ has infinite energy, like in (2), one minimizes $-2(\mu, \nu)+\|\nu\|_{e}^{2}$ instead of $\|\mu-\nu\|_{e}^{2}$, which can always be given a meaning. Cf. [63], for example.

By choosing

$$
\varphi(\zeta)= \pm \log |z-\zeta|
$$

in equation (1), the plus sign allowed for all $z \in \mathbb{C}$, the minus sign allowed only for $z \notin \Omega$, one gets the following statements for potentials:

$$
\left\{\begin{array}{c}
U^{\mu} \geq U^{\Omega} \quad \text { in all } \quad \mathbb{C} \\
U^{\mu}=U^{\Omega} \quad \text { outside } \quad \Omega .
\end{array}\right.
$$

Here

$$
U^{\mu}(z)=\frac{1}{2 \pi} \int \log \frac{1}{|z-\zeta|} d \mu(\zeta)
$$

denotes the logarithmic potential of the measure $\mu$, and $U^{\Omega}=U^{\chi_{\Omega} \cdot \mathrm{dA}}$. In particular, the measures $\mu$ and $\chi_{\Omega} \cdot(\mathrm{dA})$ are graviequivalent outside $\Omega$. By an approximation argument, (3) is actually equivalent to (1). 
In order to keep the treatment at an algebraic level we shall mainly discuss measures $\mu$ of the form (2). It can be shown [2] (see also section (3) below) that $\partial \Omega$ in this case is an algebraic curve with an equation

$$
Q(z, \bar{z})=0, \quad z \in \partial \Omega
$$

where $Q(z, w)$ is a polynomial of degree $n$ in each of $z$ and $w$. After a normalization, setting the coefficient of $z^{n} w^{n}$ equal to one, $Q$ is uniquely determined and Hermitian symmetric: $Q(z, w)=\overline{Q(\bar{w}, \bar{z})}$. However, $Q$ seems to depend in a rather transcendental way on the data $\left(a_{j}, c_{j}\right)$, and no general method is known for efficiently finding $Q$ from these. Only in some special cases, with symmetries and small values of $n$, there are known procedures [18], [10]. Note that $Q$ contains much more data than $\mu$ (the numbers of parameters are of orders of magnitude $n^{2}$ and $n$, respectively).

Let $K=\operatorname{conv} \operatorname{supp} \mu$ be the convex hull of the support of $\mu$, i.e., the convex hull of the points $a_{1}, \ldots, a_{n}$. As mentioned, $\Omega$ can be thought of as smashed out version of $\bigcup_{j=1}^{n} B\left(a_{j}, r_{j}\right)$. The geometry of $\Omega$ which this enforces is expressed in the following sharp result.

Theorem 2.1 [28], [29], [30] Assume that $\Omega$ satisfies (1) for a measure $\mu \geq 0$ of the form (2). Then

(i) $\partial \Omega$ may have singular points (cusps, double points, isolated points), but they are all located inside $K$. Outside $K, \partial \Omega$ is smooth algebraic.

For $z \in \partial \Omega \backslash K$, let $N_{z}$ denote the inward normal of $\partial \Omega$ at $z$ (well defined by (i)).

(ii) For each $z \in \partial \Omega \backslash K, N_{z}$ intersects $K$.

(iii) For $z, w \in \partial \Omega \backslash K, z \neq w, N_{z}$ and $N_{w}$ do not intersect each other before they reach $K$. Thus $\Omega \backslash K$ is fibered by (is the disjoint union of) the inward normals from $\partial \Omega \backslash K$.

(iv) There exist $r(z)>0$ for $z \in K \cap \Omega$ such that

$$
\Omega=\bigcup_{z \in K \cap \Omega} B(z, r(z))
$$

(Statement (iv) is actually a consequence of (iii).)

Remark 2.2 The theorem remains true for any measure $\mu \geq 0$ with compact support, with "analytic" in place of "algebraic" in $(i)$.

Since $\Omega$ is uniquely determined by $\left(a_{j}, c_{j}\right)$ one can steer $\Omega$ by changing the $c_{j}$ (or $a_{j}$ ). Such deformations are of Hele-Shaw type, as can be seen by the following computation, which applies in more general situations: Hele-Shaw 
evolution $\Omega(t)$ corresponding to a point source at $a \in \mathbb{C}$ ("injection of fluid" at a) means that $\Omega(t)$ changes by $\partial \Omega(t)$ moving in the outward normal direction with speed

$$
-\frac{\partial G_{\Omega(t)}(\cdot, a)}{\partial n}
$$

Here $G_{\Omega}(z, a)$ denotes the Green function of the domain $\Omega$. If $\varphi$ is subharmonic in a neighborhood of $\overline{\Omega(t)}$ then, as a consequence of $G_{\Omega}(\cdot, a) \geq 0, G_{\Omega}(\cdot, a)=0$ on $\partial \Omega$ and $-\Delta G_{\Omega}(\cdot, a)=\delta_{a}$,

$$
\begin{gathered}
\frac{d}{d t} \int_{\Omega(t)} \varphi \mathrm{dA}= \\
\left.\int_{\partial \Omega(t)} \text { (speed of } \partial \Omega(t) \text { in normal direction }\right) \varphi d s \\
=-\int_{\partial \Omega(t)} \frac{\partial G_{\Omega(t)}(\cdot, a)}{\partial n} \varphi d s= \\
-\int_{\partial \Omega(t)} \frac{\partial \varphi}{\partial n} G_{\Omega(t)}(\cdot, a) d s \\
-\int_{\Omega(t)} \varphi \Delta G_{\Omega(t)}(\cdot, a) \mathrm{dA} \\
+\int_{\Omega(t)} G_{\Omega(t)}(\cdot, a) \Delta \varphi \mathrm{dA} \geq \varphi(a) .
\end{gathered}
$$

Hence, integrating from $t=0$ to an arbitrary $t>0$,

$$
\int_{\Omega(t)} \varphi \mathrm{dA} \geq \int_{\Omega(0)} \varphi \mathrm{dA}+t \varphi(a),
$$

telling that if $\Omega(0)$ is a quadrature domain for $\mu$ then $\Omega(t)$ is a quadrature domain for $\mu+t \delta_{a}$.

We mention (tangentially to this survey) that there is a variety of unsolved problems pertaining to generalized quadrature domains, in the sense that the quadrature distribution is not necessarily supported by a compact subset of the domain. Physically, the simplest of these problems describes equilibrium shapes of air bubbles or droplets of conducting fluids in the presence of an electrostatic fields. These investigations have started in the '50s in the works of Garabedian, Lewy, Schiffer, McCleod and have been recently continued by Solynin, Vassilev, Khavinson, Beneteau and others. For an extensive list of related bibliography, see [39], [6]. 


\section{Algebraic domains}

It does not make sense to allow $c_{j}<0$ in (1), (2), because $\varphi(z)=\log \left|z-a_{j}\right|$ is subharmonic and integrable in any bounded domain, and $\varphi\left(a_{j}\right)=-\infty$, so (1) will never hold with $c_{j}<0$ for some $j$.

However, if one restricts to harmonic test functions it makes good sense to allow $c_{j}<0$. Alternatively, one may consider analytic test functions and allow $c_{j} \in \mathbb{C}$ or, slightly more generally, allow $\mu$ to be an arbitrary complex-valued distribution with support in finite number of points. This can still be thought of being represented by (2), incorporating limit cases when some of the $a_{j}$ coincide. In the above cases the inequality (1) becomes equality:

$$
\int \varphi \mathrm{d} \mu=\int_{\Omega} \varphi \mathrm{dA} .
$$

On the other hand, $\Omega$ will not always be uniquely determined by $\mu$. However, $\partial \Omega$ remains algebraic, and quadrature domains as above for analytic functions are in physics literature (starting with [78]) often referred to as algebraic domains. There are good reasons for this terminology, as we shall now explain.

Given any $\mu$ as in (2) and any open set $\Omega$ containing $\operatorname{supp} \mu$, define (as distributions in all $\mathbb{C}$ )

Then

$$
\begin{gathered}
u=U^{\mu}-U^{\Omega}, \\
S(z)=\bar{z}-4 \frac{\partial u}{\partial z} .
\end{gathered}
$$

$$
\Delta u=\chi_{\Omega}-\mu, \quad \frac{\partial S}{\partial \bar{z}}=1-\chi_{\Omega}+\mu .
$$

In particular, $S(z)$ is meromorphic in $\Omega$.

It is clear from (3) that $\Omega$ is a subharmonic quadrature domain for $\mu$ if and only if $u \geq 0$ everywhere and $u=0$ outside $\Omega$. Then also $\nabla u=0$ outside $\Omega$. Similarly, the criterion for $\Omega$ being a quadrature domain for harmonic functions is that merely $u=\nabla u=0$ on $\mathbb{C} \backslash \Omega$. (The vanishing of the gradient is a consequence of the vanishing of $u$, except at certain singular points on the boundary.) To be a quadrature domain for analytic functions it is enough that just the gradient vanishes, or better in the complex-valued case, that $\frac{\partial u}{\partial z}=0$ on $\mathbb{C} \backslash \Omega$ (or just on $\partial \Omega$ ).

Thus $\Omega$ is a quadrature domain for analytic functions (an algebraic domain) if and only if

$$
S(z)=\bar{z} \quad \text { on } \partial \Omega .
$$

This means that $S(z)$ is the Schwarz function [12], [72] of $\partial \Omega$. Since $S(z)$ is meromorphic in $\Omega$ this relationship can be interpreted as saying that $z$ and 
$S(z)$ fit together to form a meromorphic functions on the Schottky double of $\Omega$, i.e., the compact symmetric Riemann surface obtained by glueing two copies of $\Omega$ together along $\partial \Omega$, one of the copies (the "backside") provided with the opposite conformal structure. The symmetry means that the surface is equipped with an anticonformal involution, namely the map taking a point on the front side to the corresponding point on the backside, and conversely.

Using the involution one actually gets one more meromorphic function. If the first one is represented as $(z, S(z))$ the other is $(S(z), z)$. Since any two meromorphic functions on a compact Riemann surface are polynomially related, it follows that there is a polynomial $Q(z, w)$ such that $Q(z, S(z))=0$ (identically). This gives (4) on the boundary.

We wish to point out that not every domain bounded by an algebraic curve is an algebraic domain in the above sense. In general, if a domain $\Omega \subset \mathbb{C}$ is bounded by an algebraic curve $Q(z, \bar{z})=0(Q$ a polynomial with Hermitian symmetry), then one can associate two compact symmetric Riemann surfaces to it: one is the Schottky double of $\Omega$ and the other is the Riemann surface classically associated to the complex curve $Q(z, w)=0$. For the latter the involution is given by $(z, w) \mapsto(\bar{w}, \bar{z})$. In the case of algebraic domains (quadrature domains for analytic functions), and only in that case, the two Riemann surfaces canonically coincide: the lifting

$$
z \mapsto(z, S(z))
$$

from $\Omega$ to the locus of $Q(z, w)=0$ extends to the Schottky double of $\Omega$ and then gives an isomorphism, respecting the symmetries, between the two Riemann surfaces.

As a simple example, the Schottky double of the simply connected domain

$$
\Omega=\left\{z=x+i y \in \mathbb{C}: x^{4}+y^{4}<1\right\}
$$

has genus zero, while the Riemann surface associated to the curve $x^{4}+y^{4}=1$ has genus 3. Hence they cannot be identified, and in fact $\Omega$ is not an algebraic domain.

Other ways of characterizing algebraic domains, by means of rational embeddings into $n$ dimensional projective space, are discussed in [24].

\section{Signed measures, instability, uniqueness}

Consider now quadrature domains (5) for harmonic test functions and realvalued measures (2). As to the relationship between the geometry of $\Omega$ and 
the location of $\operatorname{supp} \mu$ there are then drastic differences between the cases of having all $c_{j}>0$ vs. having no restrictions on the signs of $c_{j}$. This is clearly demonstrated in the following theorem due to M. Sakai [70], [71]. The second part of the theorem is discussed (and proved) in some other forms also in [16], [20], [4], [5], [85], for example.

Theorem 4.1 Let $r$ and $R$ be positive numbers, $R \geq 2 r$. Consider measures $\mu$ of the form (2) with $c_{j}$ real and related to $r$ and $R$ by

$$
\begin{aligned}
& \operatorname{supp} \mu \subset B(0, r), \\
& \sum_{j=1}^{n} c_{j}=\pi R^{2}
\end{aligned}
$$

(i) If $\mu \geq 0$, then any quadrature domain $\Omega$ for harmonic functions for $\mu$ is also a quadrature domain for subharmonic functions. Hence theorem 2.1 applies, and in addition

$$
B(0, R-r) \subset \Omega \subset B(0, R+r) .
$$

(The upper bound is actually a consequence of (iv) of theorem 2.1.)

(ii) With $\mu$ not necessarily $\geq 0$, and with no restrictions on $\sum_{j=1}^{n}\left|c_{j}\right|$ and $n$, any bounded domain containing $B(0, r)$ and having area $\pi R^{2}$ can be uniformly approximated by quadrature domains for harmonic functions for measures $\mu$ satisfying (6), (7).

Remark 4.2 The theorem remains valid (with minor adjustments of the formulation) for arbitrary measures (not necessarily of the form (2)), and also in higher dimension.

With $\mu$ a signed measure of the form (2) we still have $\sum_{j=1}^{n} c_{j}=|\Omega|$, but $\sum_{j=1}^{n}\left|c_{j}\right|$ may be much larger. In view of the theorem, the ratio

$$
\rho=\frac{\sum_{j=1}^{n} c_{j}}{\sum_{j=1}^{n}\left|c_{j}\right|}=\frac{\int d \mu}{\int|d \mu|}
$$

$(0<\rho \leq 1)$ might give an indication of how strong is the coupling between the geometry of $\operatorname{supp} \mu$ and the geometry of $\Omega$.

As mentioned, a quadrature domain for harmonic functions is not always uniquely determined by its measure $\mu$. Still there is uniqueness at the infinitesimal level, in a neighbourhood of a domain with smooth boundary: if

$$
\sum_{j=1}^{n} c_{j} \varphi\left(a_{j}\right)=\int_{\Omega} \varphi \mathrm{dA}
$$


and (for example) the $a_{j}$ are kept fixed, then one can always increase the $c_{j}$ and get a unique evolution of $\Omega$ (well-posed Hele-Shaw). This evolution may be continued indefinitely, but during the evolution $\Omega$ may undergo changes of connectivity. If $\partial \Omega$ has no singularities then one can also decrease the $c_{j}$ slightly and have a unique evolution (backward Hele-Shaw, which is ill-posed). Therefore it makes sense, in the smooth case, to write

$$
\Omega=\Omega\left(c_{1}, \ldots, c_{n}\right)
$$

for $c_{j}$ in some interval around the original values. Note however that decreasing the $c_{j}$ makes the ratio $\rho$ decrease, indicating a loss of control or stability.

In case $\partial \Omega$ does contain singular points, several scenarios are possible: the $c_{j}$ cannot be shrinked at all (e.g., if there are 3/2-cusps), the $c_{j}$ can be decreased and the evolution of $\Omega$ is unique or the $c_{j}$ can be decreased with several possible evolutions for $\Omega$ (branching). In the latter case $\Omega$ is not uniquely determined by the $c_{j}$ in a neighbourhood of the original domain, and this occurs when the singular point is a so-called special point (see section 10.4) which has arisen as the eventual stage of a hole which has been filled in an opposite evolution (with increasing $c_{j}$ ); in that case the branching (in the ill-posed direction) amounts to the choice between letting the hole appear again or just ignoring that there has ever been a hole. A specific example can be distilled from the example in section 10.4 .

For decreasing $c_{j}$, the evolution $\Omega\left(c_{1}, \ldots, c_{n}\right)$ (unique or not) always breaks down by singularity development of $\partial \Omega$ or by $\partial \Omega$ reaching some of the points $a_{j}$ (see e.g. [36], [35], [69], [32]) before $\Omega$ is empty, except in the case that $\Omega\left(c_{1}, \ldots, c_{n}\right)$ is a quadrature domain for subharmonic functions. In the latter case the $c_{j}$ (necessarily positive) can be decreased down to zero, and $\Omega$ will be empty in the limit $c_{1}=\ldots=c_{n}=0$. However, it may happen that $\Omega\left(c_{1}, \ldots, c_{n}\right)$ breaks up into components under the evolution.

In the simply connected case, $\Omega$ will be the image of the unit disc $\mathbb{D}$ under a rational conformal map $f=f_{\left(c_{1}, \ldots, c_{n}\right)}: \mathbb{D} \rightarrow \Omega\left(c_{1}, \ldots, c_{n}\right)$. This rational function is simply the conformal pull-back of the meromorphic function $(z, S(z))$ on the Schottky double of $\Omega$ to the Schottky double of $\mathbb{D}$, the latter being identified with the Riemann sphere. It follows that the poles of $f$ are the mirror points (with respect to the unit circle) of the points $f^{-1}\left(a_{j}\right)$. When the $c_{j}$ increase then the $\left|f^{-1}\left(a_{j}\right)\right|$ decrease (this follows by an application of Schwarz' lemma to $f_{\text {larger } c_{j}}^{-1} \circ f_{\text {original }} c_{j}$ ), hence the poles of $f$ move away from the unit circle. Conversely, the poles of $f$ approach the unit circle as the $c_{j}$ decrease, also indicating a loss of stability.

When $\Omega$ is simply connected, the analytic and harmonic functions are equivalent as test classes for (8). In the limit case that all the points $a_{j}$ coincide, 
say $a_{1}=\ldots=a_{n}=0$, then (8) corresponds to

$$
\sum_{j=1}^{n} c_{j} \varphi^{(j-1)}(0)=\int_{\Omega} \varphi \mathrm{dA}
$$

for $\varphi$ analytic. The $c_{j}$ (allowed to be complex) now have a slightly different meaning than before. In fact, they are essentially the analytic moments of $\Omega$ :

$$
c_{j}=\frac{1}{(j-1) !} \int_{\Omega} z^{j-1} d A \quad(j=1, \ldots, n) .
$$

The higher order moments vanish, and the conformal map $f=f_{\left(c_{1}, \ldots, c_{n}\right)}$ : $\mathbb{D} \rightarrow \Omega\left(c_{1}, \ldots, c_{n}\right)$ is a polynomial of degree $n$. A precise form of the local bijectivity of the map $\left(c_{1}, \ldots, c_{n}\right) \mapsto \Omega\left(c_{1}, \ldots, c_{n}\right)$ has been established by O. Kouznetsova and V. Tkachev [40], [76], who proved an explicit formula for the (nonzero) Jacobi determinant of the map from the coefficients of $f$ to the moments $\left(c_{1}, \ldots, c_{n}\right)$ ). This formula was conjectured (and proved in some special cases) by C. Ullemar [77].

On the global level, it does not seem to be known whether (9), or (8), with a given left part, can hold for two different simply connected domains and all analytic $\varphi$.

Leaving the realm of quadrature domains, an explicit example of two different simply connected domains having the same analytic moments has been given by M. Sakai [64]. The idea of the example is that a disc and a concentric annulus of the same area have equal moments. If the disc and annulus are not concentric, then the union of them (if disjoint) will have the same moments as the domain obtained by interchanging their roles. Arranging everything carefully, with removing and adding some common parts, two different Jordan domains having equal analytic moments can be obtained. Similar examples were known earlier by A. Celmins [9], and probably even by P. S. Novikov. On the positive side, a classical theorem of Novikov [50] asserts that domains which are starshaped with respect to one and the same point are uniquely determined by their moments. See [86] for further discussions.

Returning now to quadrature domains, there is definitely no uniqueness for harmonic and analytic test classes if multiply connected domains are allowed. If $\Omega$ has connectivity $m+1(m \geq 1)$, i.e., has $m$ "holes", then there is generically an $m$-parameter family $\Omega\left(t_{1}, \ldots, t_{m}\right)$ of domains such that $\Omega(0, \ldots, 0)=$ $\Omega$ and

$$
\frac{\partial}{\partial t_{j}} \int_{\Omega\left(t_{1}, \ldots, t_{m}\right)} \varphi \mathrm{dA}=0 \quad(j=1, \ldots, m)
$$

for every $\varphi$ analytic in a neighborhood of the domains. (See section 10.4 for an example of a one-parameter family.) These deformations are Hele-Shaw 
evolutions, driven not by Green functions but by "harmonic measures", i.e., regular harmonic functions which take (different) constant boundary values on the components of $\partial \Omega$.

It follows that multiply connected quadrature domains for analytic functions for a given $\mu$ occur in continuous families. It even turns out [19], [73] that any two algebraic domains for the same $\mu$ can be deformed into each other through families as above. Thus there is a kind of uniqueness at a higher level: given any $\mu$ there is at most one connected family of algebraic domains belonging to it.

For harmonic quadrature domains there are no such continuous families (choosing $\varphi(z)=\log |z-a|$ in (8) with $a \in \mathbb{C} \backslash \bar{\Omega}$ in the holes stops them), but one can still construct examples with a discrete set of different domains for the same $\mu$. It is for example possible to imitate the example with a disc and an annulus with quadrature domains for measures $\mu$ of the form (2), with $a_{j}=e^{2 \pi j / n}(n \geq 3)$ and $c_{1}=\ldots=c_{n}=c>0$ suitably chosen. See [18], [10], and section 10.4 below. However, it seems very difficult to imitate the full Sakai construction, with "removing and adding some common parts", in the context of quadrature domains. Therefore it is not at all easy to construct different simply connected quadrature domains for the same $\mu$.

\section{Markov's moment problem}

We open this new section with a discussion of a second, rather independent perspective on quadrature domains.

In his investigations of the limiting values of certain integrals appearing in probability theory A. A. Markov has isolated a truncated moment problem with bounds. It was M. G. Krein who put Markov's problem into an abstract setting, and raised it to the level of a simple and very flexible principle of duality on locally convex spaces $[3,41]$. It is in this framework that various finite determinateness phenomena, including among these the dependence of quadrature domains on a finite set of their moments, find their natural explanation ( see in addition [38]). We confine ourselves below to a generic form of Krein's interpretation of Markov moment problem.

Let $(X, \Sigma, \mu)$ be a measure space, with $\mu$ a positive, finite mass measure. Let $f_{1}, \ldots, f_{n} \in L^{1}(X, \mu)$ be a finite, linearly independent (over $\mathbb{R}$ ) system of (real valued) integrable functions, such that the level set of any nontrivial linear combination has zero mass:

$$
\mu\left\{x \in X ; \lambda_{1} f_{1}+\ldots+\lambda_{n} f_{n}=\gamma\right\}=0, \quad \gamma \in \mathbb{R}
$$


Markov's moment problem consists of a finite numerical data $\left(c_{1}, \ldots, c_{n}\right) \in \mathbb{R}^{n}$ and an unknown function $g \in L^{\infty}(X)$, subject to the generalized moments and bound conditions:

$$
\int f_{j} g d \mu=c_{j}, \quad 1 \leq j \leq n ; \quad\|g\|_{\infty} \leq 1
$$

The main result can be stated as follows, see for more details [41].

Theorem 5.1 There exists a function $g \in L^{\infty}(X)$ satisfying (10) if and only if

$$
\sum_{j=1}^{n} \lambda_{j} c_{j} \leq \int\left|\sum_{j=1}^{n} \lambda_{j} f_{j}\right| d \mu
$$

for all scalars $\lambda_{j}$.

Moreover, the solution $g$ is unique (up to a modification on a null set) if and only if there are scalars $\tilde{\lambda}_{j}$, such that the above inequality is an equality, and in this case

$$
g=\operatorname{sgn} \sum_{j=1}^{n} \tilde{\lambda}_{j} f_{j}
$$

The proof of the first sentence is an application of Hahn-Banach Theorem, remarking that the linear functional $L$ which maps $f_{j}$ into $c_{j}$ has norm at most one if and only if the inequality in the statement holds. If the inequality is strict, obviously there are infinitely many solutions of the form $g^{\prime}=g+\epsilon h$ where $\int f_{j} h d \mu=0$ for all $j$ and $\epsilon$ is sufficiently small.

Assume that the inequality is an equality:

$$
\int\left(\sum_{j=1}^{n} \tilde{\lambda}_{j} f_{j}\right) g d \mu=\int\left|\sum_{j=1}^{n} \tilde{\lambda}_{j} f_{j}\right| d \mu .
$$

Since $-1 \leq g \leq 1$ we infer that the function $g$ has only two values, \pm 1 , and as a matter of fact it coincides with the signum of $\sum_{j=1}^{n} \tilde{\lambda}_{j} f_{j}$.

A typical application would require a fixed frame, say a closed cube $\Delta$ in $\mathbb{R}^{n}$ and a fixed degree $d$. We consider as test functions all monomials $x^{\alpha},|\alpha| \leq$ $d$, of degree less than or equal to $d$ (adopting throughout the multi-index notation). Then Markov's problem:

$$
\int_{\Delta} g(x) x^{\alpha} d x=c_{\alpha}, \quad|\alpha| \leq d
$$

admits a measurable function $g: \Delta \longrightarrow[-1,1]$ as solution if and only if for 
all polynomials $p(x)=\sum_{|\alpha| \leq d} a_{\alpha} x^{\alpha}$, the inequality:

$$
\sum_{|\alpha| \leq d} a_{\alpha} c_{\alpha} \leq \int_{\Delta}|p(x)| d x
$$

holds. Quite remarkably, this problem has a unique solution if and only if there exists a polynomial $q$ of degree less than or equal to $d$, such that

$$
c_{\alpha}=\int_{\{q(x)>0\} \cap \Delta} x^{\alpha} d x-\int_{\{q(x)<0\} \cap \Delta} x^{\alpha} d x, \quad|\alpha| \leq d .
$$

Thus, in this extremal case, the recovery of the original "shade" function $q$ from its moments

$$
\left\{c_{\alpha} ;|\alpha| \leq d\right\} \longrightarrow q(x)
$$

is well posed. However, the constructive aspects of this unique determination remain unclear in general.

Next we will show that quadrature domains fit into this scheme and well illustrate a simple and robust recovery algorithm.

\section{The trace formula}

A normal operator is modelled via the spectral theorem as multiplication by the complex variable on a vector valued Lebesgue $L^{2}$-space. The interplay between measure theory and the structure of normal operators is well known and widely used in applications. One step further, there are by now well understood classes of close to normal operators; their classification and functional models are sensibly more involved, but not less interesting, see [46,81]. We record below a few aspects of the theory of semi-normal operators with trace class self-commutators. They will be significant for our survey of quadrature domains.

Let $H$ be a separable, complex Hilbert space and let $T \in \mathcal{L}(H)$ be a linear bounded operator. We assume that the self-commutator $\left[T^{*}, T\right]=T^{*} T-T T^{*}$ is trace-class. Then for a pair of polynomials $p(z, \bar{z}), q(z, \bar{z})$ one can choose an ordering in the functional calculus $p\left(T, T^{*}\right), q\left(T, T^{*}\right)$. The functional

$$
(p, q) \rightarrow \operatorname{trace}\left[p\left(T, T^{*}\right), q\left(T, T^{*}\right)\right]
$$

is then well-defined, independent of the ordering in the functional calculus,

and possesses the algebraic identities of the Jacobian $\frac{\partial(p, q)}{\partial(\bar{z}, z)}$. Thus, a direct 
reasoning will imply the existence of a distribution $u_{T} \in \mathcal{D}^{\prime}(\mathbb{C})$ satisfying

$$
\operatorname{trace}\left[p\left(T, T^{*}\right), q\left(T, T^{*}\right)\right]=u_{T}\left(\frac{\partial(p, q)}{\partial(\bar{z}, z)}\right)
$$

see [34]. The distribution $u_{T}$ exists in any number of variables (that is for tuples of self-adjoint operators subject to a trace class multi-commutator condition) and it is known as the Helton-Howe functional.

Dimension two is special because of a theorem of J. D. Pincus which asserts that $u_{T}=\frac{1}{\pi} g_{T} \mathrm{dA}$, that is $u_{T}$ is given by an integrable function function $g_{T}$, called the principal function of the operator $T$, see $[51,8]$. In particular, the moments of the function $g_{T}$ have a simple Hilbert space interpretation:

$$
\begin{aligned}
& m k \int z^{m-1} \bar{z}^{k-1} g_{T}(z) \mathrm{dA}= \\
& \operatorname{trace}\left[T^{* k}, T^{m}\right], \quad k, m \geq 1 .
\end{aligned}
$$

In general, the principal function can be regarded as a generalized Fredholm index of $T$ : when the left hand side is well defined, we have

$$
\operatorname{ind}(T-\lambda)=-g_{T}(\lambda)
$$

Moreover $g_{T}$ enjoys the functoriality properties of the index, and it is obviously invariant under trace class perturbations of $T$.

A simple, yet non-trivial, example can be constructed as follows. Let $\Omega$ be a planar domain bounded by a smooth Jordan curve $\Gamma$. Let $H^{2}(\Gamma)$ be the closure of complex polynomials in the space $L^{2}(\Gamma, d s)$, where $d s$ stands for the arc length measure along $\Gamma$ (the so-called Hardy space attached to $\Gamma$ ). The elements of $H^{2}(\Gamma)$ extend analytically to $\Omega$. The multiplication operator by the complex variable, $T_{z} f=z f, f \in H^{2}(\Gamma)$, is obviously linear and bounded. The regularity assumption on $\Gamma$ implies that the commutator $\left[T_{z}, T_{z}^{*}\right]$ is trace class. Moreover, the associated principal function is the characteristic function of $\Omega$, so that the trace formula above becomes:

$$
\begin{gathered}
\operatorname{trace}\left[p\left(T_{z}, T_{z}^{*}\right), q\left(T_{z}, T_{z}^{*}\right)\right]= \\
\frac{1}{\pi} \int_{\Omega}\left(\frac{\partial(p, q)}{\partial(\bar{z}, z)}\right) \mathrm{dA}, \quad p, q \in \mathbb{C}[z, \bar{z}] .
\end{gathered}
$$

See for details $[46,81]$. 


\section{$7 \quad$ A matrix model for quadrature domains}

In general, the principal function does not determine the original operator. The uniqueness is restored when working with the specific class of rank-one self- commutator operators. To be more specific we assume that $T \in \mathcal{L}(H)$ is irreducible (i.e. $T$ is not a direct sum of two operators) and satisfies

$$
\left[T^{*}, T\right] h=\xi\langle h, \xi\rangle, \quad h \in H,
$$

where the vector $\xi$ is fixed. Then the associated principal function $g_{T}$ becomes a complete unitary invariant for $T$. Moreover, any measurable function of compact support and having real values between $[0,1]$ can be the principal function of such an operator, see [51]. In this case the spectrum $\sigma(T)$ of $T$ is equal to the closed support of $g_{T}$, and many other refined spectral properties of $T$ can be read from $g_{T}$, see again for details $[46,81]$.

Besides the trace formula, a canonical determinant formula (going back to the works of Pincus and Larry Brown [51,7]) holds in this case:

$$
\begin{gathered}
\operatorname{det}\left[(T-z)\left(T^{*}-\bar{z}\right)(T-z)^{-1}\left(T^{*}-\bar{z}\right)^{-1}\right]= \\
1-\left\|\left(T^{*}-\bar{z}\right)^{-1} \xi\right\|^{2}= \\
\exp \left[\frac{-1}{\pi} \int_{\mathbb{C}} \frac{g_{T}(w) \mathrm{dA}(w)}{(w-z)(\bar{w}-\bar{z})}\right], \quad z \notin \sigma(T) .
\end{gathered}
$$

In conclusion, we have a constructive correspondence between all measurable functions of compact support $g_{T}: \mathbb{C} \longrightarrow[0,1]$ and all irreducible operators $T$ with rank-one self-commutator. The class of (characteristic functions) of quadrature domains stands aside in this correspondence.

Theorem 7.1 ([55]) Let $T$ be an irreducible operator with rank-one selfcommutator $\left[T^{*}, T\right]=\xi\langle\cdot, \xi\rangle$ and principal function $g_{T}$. Then $g_{T}$ is the characteristic function of a quadrature domain $\Omega$ if and only if the linear span $\bigvee_{k \geq 0} T^{* k} \xi$ is finite dimensional.

This result offers an efficient characterization of quadrature domains in terms of a finite set of their moments (see the reconstruction section below) and it opens a new link between quadrature domains and linear algebra. We only describe a few results in this direction. For more details see $[23,24,55,56]$.

In the conditions of the above Theorem, let $H_{0}=\bigvee_{k \geq 0} T^{* k} \xi$ and let $p$ denote the orthogonal projection of the Hilbert space $H$ (where $T$ acts) onto $H_{0}$. Denote $C_{0}=p T p$ (the compression of $T$ to the $d$-dimensional space $H_{0}$ ) and 
$D_{0}^{2}=\left[T^{*}, T\right]$. Then the operator $T$ has a two block-diagonal structure:

$$
T=\left(\begin{array}{ccccc}
C_{0} & 0 & 0 & 0 & \ldots \\
D_{1} & C_{1} & 0 & 0 & \ldots \\
0 & D_{2} & C_{2} & 0 & \ldots \\
0 & 0 & D_{3} & C_{3} & \ldots \\
\vdots & & \vdots & & \ddots
\end{array}\right),
$$

where the entries are all $d \times d$ matrices, recurrently defined by the system of equations:

$$
\left\{\begin{array}{l}
{\left[C_{k}{ }^{*}, C_{k}\right]+D_{k+1}{ }^{*} D_{k+1}=D_{k} D_{k}{ }^{*}} \\
C_{k+1}{ }^{*} D_{k+1}=D_{k+1} C_{k}{ }^{*}, \quad k \geq 0 .
\end{array}\right.
$$

Note that $D_{k}>0$ for all $k$. This decomposition has an array of consequences:

(1) The spectrum of $C_{0}$ coincides with the quadrature nodes of $\Omega$;

(2) $\Omega=\left\{z ;\left\|\left(C_{0}^{*}-\bar{z}\right)^{-1} \xi\right\|>1\right\}$ (up to a finite set);

(3) The quadrature identity becomes

$$
\int_{\Omega} f(z) \mathrm{dA}(z)=\pi\left\langle f\left(C_{0}\right) \xi, \xi\right\rangle,
$$

for $f$ analytic in a neighborhood of $\bar{\Omega}$;

(4) The Schwarz function of $\Omega$ is

$$
S(z)=\bar{z}-\left\langle\xi,\left(C_{0}^{*}-\bar{z}\right)^{-1} \xi\right\rangle+\left\langle\xi,\left(T^{*}-\bar{z}\right)^{-1} \xi\right\rangle,
$$

where $z \in \Omega$.

To give the simplest and most important example, let $\Omega=\mathbb{D}$ be the unit disk (which is a quadrature domain of order one). Then the associated operator is the unilateral shift $T=T_{z}$ acting on the Hardy space $H^{2}(\partial \mathbb{D})$. Denoting by $z^{n}$ the orthonormal basis of this space we have $T z^{n}=z^{n+1}, \quad n \geq 0$, and $\left[T^{*}, T\right]=1\langle\cdot, 1\rangle$ is the projection onto the first coordinate $1=z^{0}$. The space $H_{0}$ is one dimensional and $C_{0}=0$. This will propagate to $C_{k}=0$ and $D_{k}=1$ for all $k$. Thus the matricial decomposition of $T$ becomes the familiar realization of the shift as an infinite Jordan block. 


\section{The exponential transform}

Let $\Omega$ be a bounded planar domain. The above determinantal formula (11) motivates a closer look at the exponential transform:

$$
E_{\Omega}(z, w)=\exp \left[\frac{-1}{\pi} \int_{\Omega} \frac{\mathrm{dA}(w)}{(w-z)(\bar{w}-\bar{z})}\right]
$$

It turns out by simple arguments that $E_{\Omega}$ is well defined and separately continuous everywhere on $\mathbb{C}^{2}$, provided the convention $\exp (-\infty)=0$ is adopted. This transform can be regarded as a limit Riesz potential $([61,62])$ and it can be extended canonically to any number of variables $([26,58])$. At the level of distributions, the following remarkable equation holds:

$$
|z-w|^{2} \frac{\partial^{2}}{\partial \bar{z} \partial w} E_{\Omega}(z, w)=-E(z, w) \chi_{\Omega}(z) \chi_{\Omega}(w)
$$

This, together with the positive definiteness of the kernel $1-E_{\Omega}(z, w)$, provides an alternate route of computing the linear data ( $T$ of the preceding section) associated to $\Omega$ and all related fine invariants, see for details [25]. The exponential transform was used as a technical tool in the proof of the regularity of certain two dimensional free boundaries [23].

The following result, a consequence of Theorem 7.1, is relevant for the present note.

Theorem 8.1 ([55]) The bounded planar domain $\Omega$ is a quadrature domain if and only if the germ of $E_{\Omega}$ at infinity is rational, of the form $E_{\Omega}(z, w)=$ $\frac{Q(z, \bar{w})}{P(z) \overline{P(w)}}$. In this case, choosing an irreducible representation, $Q(z, \bar{z})=0$ is the defining equation of $\partial \Omega$ and $P(z)$ is the monic polynomial vanishing at the quadrature nodes of $\Omega$.

For instance, if $\Omega=\mathbb{D}$ is the unit disk, then

$$
E_{\mathbb{D}}(z, w)=\frac{z \bar{w}-1}{z \bar{w}}
$$

In view of the linear algebra realization outlined in the preceding section we obtain more information about the defining equation of the quadrature domain. For instance:

$$
\frac{Q(z, \bar{w})}{P(z) \overline{P(w)}}=1-\left\langle\left(C_{0}^{*}-\bar{w}\right)^{-1} \xi,\left(C_{0}^{*}-\bar{z}\right)^{-1} \xi\right\rangle
$$


which yields

$$
Q(z, z)=|P(z)|^{2}-\sum_{k=0}^{d-1}\left|Q_{k}(z)\right|^{2},
$$

where $Q_{k}$ is a polynomial of degree $k$ in $z$, see [24].

Assuming that the quadrature nodes $\left\{a_{1}, \ldots, a_{d}\right\}$ are simple we have $P(z)=$ $\left(z-a_{1}\right) \ldots\left(z-a_{d}\right)$ and consequently a simple fraction decomposition is available:

$$
E_{\Omega}(z, \bar{w})=1-\sum_{j, k=1}^{d} \frac{h_{j k}}{\left(z-a_{j}\right)\left(\bar{w}-\overline{a_{k}}\right)},
$$

where the matrix

$$
h_{j k}=-\frac{Q\left(a_{j}, \overline{a_{k}}\right)}{P^{\prime}\left(a_{j}\right) \overline{P^{\prime}\left(a_{k}\right)}},
$$

is positive semi-definite.

\section{A reconstruction algorithm}

At this stage we can return, with more information and better tools, to Markov's moment problem.

Let $\left(a_{m n}\right)_{m, n=0}^{d}$ be a finite moment sequence of a measurable function of compact support $g: \mathbf{C} \longrightarrow[0,1]$ :

$$
a_{m n}=\int_{\mathbb{C}} z^{n} \bar{z}^{m} g(z) \mathrm{d} \operatorname{Area}(z) .
$$

Let us consider its formal exponential transform:

$$
\exp \left[\frac{-1}{\pi} \sum_{m, n=0}^{d} \frac{a_{m n}}{z^{n+1} \bar{w}^{m+1}}\right]=1-\sum_{m, n=0}^{\infty} \frac{b_{m n}}{z^{n+1} \bar{w}^{m+1}} .
$$

A characterization of all sequences $\left(b_{m n}\right)$ which can occur in this process is readily available from the operator theory interpretation, see [46]. Moreover, Theorem (7.1) gives a simple characterization of $g=\chi_{\Omega}$, where $\Omega$ is a quadrature domain. We put this information into the form of a recovery algorithm:

(1) Assume that $\operatorname{det}\left(b_{m n}\right)_{m, n=0}^{d}=0$ and that $d$ is the minimal integer with this property, that is $\operatorname{det}\left(b_{m n}\right)_{m, n=0}^{d-1} \neq 0$. Solve the system:

$$
\sum_{m=0}^{d} b_{m n} c_{m}=0,0 \leq n \leq d
$$


with the normalization $c_{d}=1$.

(2) Consider the polynomial $P(z)=c_{d} z^{d}+c_{d-1} z^{d-1}+\ldots+c_{0}$ and isolate from the following product the polynomial part $Q$ :

$$
\begin{gathered}
P(z) \overline{P(w)} \sum_{m, n=0}^{d} \frac{b_{m n}}{z^{n+1} \bar{w}^{m+1}}= \\
Q(z, \bar{w})+O\left(\frac{1}{z}, \frac{1}{\bar{w}}\right) .
\end{gathered}
$$

(3) The function $g$ equals, up to a null set, the characteristic function of the quadrature domain $\Omega=\{z \in \mathbf{C} ; Q(z, \bar{z})<0\}$.

In the case of more general domains, but still finitely many moment data, one replaces the second step by a variational problem:

Find $\left(c_{0}=1, c_{1}, \ldots, c_{d}\right)$ which minimizes the Hermitian form

$$
\sum_{m=0}^{d} b_{m n} c_{m} \overline{c_{n}} .
$$

Then the equation $Q(z, \bar{z})<0$ approximates $\Omega$.

Step three of the algorithm is a two dimensional analogue of a diagonal Padé approximation scheme, see for details [57]. An analysis of the convergence of the algorithm is contained in [22], while some applications to image processing are discussed in $[22,15]$. It is worth mentioning that standard tomographic data can be translated into finitely many moments, and viceversa, see [15].

\section{Examples}

\subsection{Reconstruction of a disk}

Start with the data:

$$
a_{00}=\pi r^{2}, a_{01}=\overline{a_{10}}=\pi a r^{2}, a_{11}=\pi|a|^{2} r^{2}+\pi r^{4},
$$

where $r>0$ and $a \in \mathbf{C}$. Its exponential transform is:

$$
\begin{gathered}
\exp \left[-\frac{r^{2}}{z \bar{z}}-\frac{a r^{2}}{z^{2} \bar{z}}-\frac{\bar{a} r^{2}}{z \bar{z}^{2}}-\frac{|a|^{2} r^{2}+r^{4}}{z^{2} \bar{z}^{2}}\right]= \\
1-\frac{r^{2}}{z \bar{z}}-\frac{a r^{2}}{z^{2} \bar{z}}-\frac{\bar{a} r^{2}}{z \bar{z}^{2}}-\frac{|a|^{2} r^{2}+r^{4} / 2}{z^{2} \bar{z}^{2}}+\frac{1}{2} \frac{z^{4}}{z^{2} \bar{z}^{2}}+\ldots,
\end{gathered}
$$


so that

$$
b_{00}=r^{2}, b_{01}=\overline{b_{10}}=a r^{2}, \quad b_{11}=|a|^{2} r^{2} .
$$

The determinant of the associated matrix vanishes:

$$
\operatorname{det}\left(b_{i j}\right)_{i, j=0}^{1}=|a|^{2} r^{4}-|a|^{2} r^{4}=0
$$

and the vector $(-a, 1)^{T}$ is annihilated by this matrix. Therefore

$$
P(z)=z-a,
$$

and

$$
\begin{gathered}
(z-a)(\bar{w}-\bar{a})\left[1-\frac{r^{2}}{z \bar{z}}-\frac{a r^{2}}{z^{2} \bar{z}}-\frac{\bar{a} r^{2}}{z \bar{z}^{2}}-\frac{|a|^{2} r^{2}}{z^{2} \bar{z}^{2}}+\ldots\right]= \\
(z-a)(\bar{w}-\bar{a})-r^{2}+O\left(\frac{1}{z}, \frac{1}{\bar{w}}\right) .
\end{gathered}
$$

Thus the domain with the prescribed moments is the disk of equation $\mid z-$ $\left.a\right|^{2}-r^{2}<0$.

\subsection{Quadrature domains with a double node}

Let $z=w^{2}+b w$ be the conformal mapping of the disk $|w|<1$, where $b \geq 2$. Then $z$ describes a quadrature domain $\Omega$ of order 2 , whose boundary has the equation:

$$
Q(z, \bar{z})=|z|^{4}-\left(2+b^{2}\right)|z|^{2}-b^{2} z-b^{2} \bar{z}+1-b^{2}=0 .
$$

The Schwarz function of $\Omega$ has a double pole at $z=0$, whence the associated $2 \times 2$-matrix $C_{0}$ (see Section 7) is nilpotent. Moreover, we know that:

$$
|z|^{4}\left\|\left(C_{0}^{*}-\bar{z}\right)^{-1} \xi\right\|^{2}=|z|^{4}-P(z, \bar{z}) .
$$

Therefore

$$
\left\|\left(C_{0}^{*}+\bar{z}\right) \xi\right\|^{2}=\left(2+b^{2}\right)|z|^{2}+b^{2} z+b^{2} \bar{z}+b^{2}-1,
$$

or equivalently: $\|\xi\|^{2}=2+b^{2},\left\langle C_{0}^{*} \xi, \xi\right\rangle=b^{2}$ and $\left\|C_{0}^{*} \xi\right\|^{2}=b^{2}-1$.

Consequently the linear data of the quadrature domain $\Omega$ are:

$$
C_{0}^{*}=\left(\begin{array}{c}
0 \frac{b^{2}-1}{\left(b^{2}-2\right)^{1 / 2}} \\
0 \\
0
\end{array}\right), \quad \xi=\left(\begin{array}{c}
\frac{b^{2}}{\left(b^{2}-1\right)^{1 / 2}} \\
\left(\frac{b^{2}-2}{b^{2}-1}\right)^{1 / 2}
\end{array}\right) .
$$




\subsection{Quadrature domains with two distinct nodes}

Assume that the nodes are fixed at \pm 1 . Hence $P(z)=z^{2}-1$. The defining equation of the quadrature domain $\Omega$ of order two with these nodes is:

$$
Q(z, \bar{z})=\left(|z+1|^{2}-r^{2}\right)\left(|z-1|^{2}-r^{2}\right)-c,
$$

where $r$ is a positive constant and $c \geq 0$ is chosen so that either $\Omega$ is a union of two disjoint open disks (in which case $c=0$ ), or $Q(0,0)=0$, see [18]. A short computation yields:

$$
Q(z, \bar{z})=z^{2} \bar{z}^{2}-2 r z \bar{z}-z^{2}-\bar{z}^{2}+\alpha(r)
$$

where

$$
\alpha(r)= \begin{cases}\left(1-r^{2}\right)^{2}, & r<1 \\ 0, & r \geq 1\end{cases}
$$

One step further, we can identify the linear data from the identity:

$$
|P(z)|^{2}\left(1-\left\|\left(C_{0}^{*}-\bar{z}\right)^{-1} \xi\right\|^{2}\right)=Q(z, \bar{z}) .
$$

Consequently,

$$
\xi=\left(\begin{array}{c}
\sqrt{2} r \\
0
\end{array}\right), C_{0}^{*}=\left(\begin{array}{cc}
0 & \frac{\sqrt{2} r}{\sqrt{1-\alpha(r)}} \\
\frac{\sqrt{1-\alpha(r)}}{\sqrt{2} r} & 0
\end{array}\right)
$$

This simple computation illustrates the fact that, although the process is affine in $r$, the linear data of the growing domains have discontinuous derivatives at the exact moment when the connectivity changes.

10.4 Three points, non-simply connected quadrature domains and the nonuniqueness phenomenon

Quadrature domains (for analytic functions) with at most two nodes, as in the above examples, are uniquely determined by their quadrature data and are simply connected. For three nodes and more it is no longer so. The following example, taken from [18], with three nodes and symmetry under rotations by $2 \pi / 3$, illustrates the general situation quite well. More details on the present example are given in [18], and similar examples with more nodes are studied in $[10]$. 
Let the quadrature nodes and weights be $a_{j}=\omega^{j}$ and $c_{j}=\pi r^{2}$ respectively $(j=1,2,3)$, where $\omega=e^{2 \pi i / 3}$ and where $r>0$ is a parameter. Considering first the strongest form of quadrature property, namely for subharmonic functions, as in (1), (2), the situation is in principle easy: $\Omega$ is for any given $r>0$ uniquely determined up to nullsets and can be viewed as a swept out version of the quadrature measure $\mu=\sum_{j=1}^{3} c_{j} \delta_{a_{j}}$ or as the union of the $\operatorname{discs} B\left(a_{j}, r\right)$ with (possible) multiple coverings smashed out.

For $0<r \leq \frac{\sqrt{3}}{2}$ the above discs are disjoint, hence $\Omega=\cup_{j=1}^{3} B\left(a_{j}, r\right)$. For $r$ larger than $\frac{\sqrt{3}}{2}$ but smaller than a certain critical value $r_{0}$ (which seems to be difficult to determine explicitly) $\Omega$ is doubly connected with a hole containing the origin, while for $r \geq r_{0}$ the hole will be filled in so that $\Omega$ is a simply connected domain. The above quadrature domains (or open sets) are actually uniquely determined even within nullsets, except in the case $r=r_{0}$ when both $\Omega$ and $\Omega \backslash\{0\}$ satisfy $(1)$.

Consider next the general class of quadrature domains for analytic functions (algebraic domains). For $0<r \leq \frac{\sqrt{3}}{2}$ only the disjoint discs qualify, as before. However, for any $r>\frac{\sqrt{3}}{2}$ there is a whole one-parameter family of domains $\Omega$ satisfying (5) for analytic $\varphi$. These are defined by the polynomials

$$
\begin{aligned}
& Q(z, \bar{z})=z^{3} \bar{z}^{3}-z^{3}-\bar{z}^{3}-3 r^{2} z^{2} \bar{z}^{2}- \\
& 3 \tau\left(\tau^{3}-2 r^{2} \tau+1\right) z \bar{z}+\tau^{3}\left(2 \tau^{3}-3 r^{2} \tau+1\right),
\end{aligned}
$$

where $\tau>0$ is a free parameter, independent of the quadrature data. When completed as to nullsets, the quadrature domains in question are more precisely

$$
\Omega(r, \tau)=\operatorname{intclos}\{z \in \mathbb{C}: Q(z, \bar{z})<0\}
$$

The interpretation of the parameter $\tau$ is that on each radius $\left\{z=t \omega^{j+\frac{1}{2}}\right.$ : $t>0\}, j=1,2,3$, there is exactly one singular point of the algebraic curve $Q(z, \bar{z})=0$, and $\tau=|z|$ for that point. This singular point is either a cusp on $\partial \Omega$ or an isolated point of $Q(z, \bar{z})=0$, a so-called special point. Special points are those points $a \in \Omega$ for which the quadrature identity (5) admits the (integrable) meromorphic function $\varphi(z)=\frac{1}{z-a}$. Equivalently, $\Omega \backslash\{a\}$ remains to be a quadrature domain for integrable analytic functions.

For $\frac{\sqrt{3}}{2}<r<2^{-\frac{1}{6}}$ the quadrature domains for analytic functions are exactly the domains $\Omega(r, \tau)$ (with possible removal of special points) for $\tau$ in an interval $\tau_{1}(r) \leq \tau \leq \tau_{2}(r)$, where $\tau_{1}(r), \tau_{2}(r)$ satisfy $0<\tau_{1}(r)<\frac{1}{2}<\tau_{2}(r)$, and more precisely can be defined as the positive zeros of the polynomial $4 \tau^{3}-4 r^{2} \tau+1$. (see [18] for further explanations and proofs). The domains $\Omega(r, \tau)$ are doubly connected with a hole containing the origin. When $\tau$ increases the hole shrinks and both boundary components move towards the origin. For $\tau=\tau_{2}(r)$ there 
are three cusps on the outer boundary component which stop further shrinking of the hole, and for $\tau=\tau_{1}(r)$ there are three cusps on the inner boundary component which stop the expansion of the hole.

For exactly one parameter value, $\tau=\tau_{\text {subh }}(r), \Omega(r, \tau)$ is a quadrature domain for subharmonic functions (and so also for harmonic functions). This $\tau_{\text {subh }}(r)$ can be determined implicitly by evaluating the quadrature identity (5) for $\varphi(z)=\log |z|$, which gives the equation

$$
\int_{\Omega\left(r, \tau_{\mathrm{subh}}(r)\right)} \log |z| \mathrm{dA}(z)=0 .
$$

For $r=\frac{\sqrt{3}}{2}, \tau_{1}(r)=\tau_{2}(r)=\frac{1}{2}$, and as $r$ increases, $\tau_{1}(r)$ decreases and $\tau_{2}(r)$ increases. What happens when $r=2^{-\frac{1}{6}}$ is that for $\Omega\left(r, \tau_{2}(r)\right)$, i.e., for the domain with cusps on the outer component, the hole has shrunk to a point (the origin). Hence, for $r=2^{-\frac{1}{6}}, \Omega\left(r, \tau_{2}(r)\right)$ is simply connected, while $\Omega(r, \tau)$ for $\tau_{1}(r) \leq \tau<\tau_{2}(r)$ remain doubly connected.

For all $\frac{\sqrt{3}}{2}<r \leq 2^{-\frac{1}{6}}, \tau_{1}(r)<\tau_{\text {subh }}(r)<\tau_{2}(r)$ because a subharmonic quadrature domain cannot have the type of cusps which appear for $\tau=\tau_{1}(r), \tau_{2}(r)$ (see [68], [69]). It follows that the critical value $r=r_{0}$, when $\Omega\left(r, \tau_{\text {subh }}(r)\right.$ ) becomes simply connected, is larger that $2^{-\frac{1}{6}}$.

For $r \geq 2^{-\frac{1}{6}}$ the quadrature domains for analytic functions are the domains $\Omega(r, \tau)$ (with possible deletion of special points), with $\tau$ in an interval $\tau_{1}(r) \leq$ $\tau \leq \tau_{3}(r)$. Here $\tau_{1}(r)$ is the same as before (i.e., corresponds to cusps on the inner boundary), while $\tau_{3}(r)$ is the value of $\tau$ for which the hole at the origin degenerates to just the origin itself (which for $r>2^{-\frac{1}{6}}$ occurs before cusps have developed on the outer boundary). The origin then is a special point, and one concludes from (13) that $\tau=\tau_{3}(r)$ is the smallest positive zero of the polynomial $2 \tau^{3}-3 r^{2} \tau+1$. For $r=2^{-\frac{1}{6}}, \tau_{3}(r)=\tau_{2}(r)=2^{-\frac{2}{3}}$.

For $2^{-\frac{1}{6}} \leq r<r_{0}$ we have $\tau_{1}(r)<\tau_{\text {subh }}(r)<\tau_{3}(r)$, while for $r \geq r_{0}$, $\tau_{\text {subh }}(r)=\tau_{3}(r)$. Since $\Omega\left(r, \tau_{3}(r)\right)$ is simply connected and is a quadrature domain for analytic functions it is also a quadrature domain for harmonic functions. It follows that in the interval $2^{-\frac{1}{6}} \leq r<r_{0}$ there are (for each $r$ ) two different quadrature domains for harmonic functions, namely $\Omega\left(r, \tau_{\text {subh }}(r)\right)$ and $\Omega\left(r, \tau_{3}(r)\right)$ (doubly respectively simply connected).

In summary, we have for each $r>\frac{\sqrt{3}}{2}$ a one-parameter family of algebraic domains $\Omega(r, \tau)$, for exactly one parameter value $\left(\tau=\tau_{\text {subh }}(r)\right)$ this is a quadrature domain for subharmonic functions, and for each $r$ in a certain interval $\left(2^{-\frac{1}{6}} \leq r<r_{0}\right)$ there are two different quadrature domains for harmonic functions $\left(\Omega\left(r, \tau_{\text {subh }}(r)\right)\right.$ and $\left.\Omega\left(r, \tau_{3}(r)\right)\right)$. 


\section{References}

[1] O. Agam, E. Bettelheim, P. Wiegmann, A. Zabrodin, Viscous fingering and the shape of an electronic droplet in the Quantum Hall regime, Phys. Rev. Letter. $\mathbf{8 8}$ (2002), 1-4.

[2] D. Aharonov and H.S. Shapiro, Domains on which analytic functions satisfy quadrature identities, J. Analyse Math., 30 (1976), 39-73.

[3] N. I. Akhiezer, M.G. Krein, Some Questions in the Theory of Moments, Amer. Math. Soc. Transl. Vol. 2 Amer. Math. Soc., Providence, R.I., 1962.

[4] S. Bell, Quadrature domains and kernel function zipping Ark. Mat. 43 (2005), 271-287.

[5] S. Bell, The Bergman kernel and quadrature domains in the plane, pp. 61-78 in [59].

[6] C. Beneteau, D. Khavinson, The isoperimetric inequality via approximation theory and free boundary problems, Comput. Methods Funct. Theory 6(2) (2006), 253-274.

[7] L. Brown, The determinant invariant for operators with trace class selfcommutator, in "Proc. Conference on Operator Theory", Lect. Notes Math. Vol. 345, Springer, Berlin, 1973.

[8] R.W. Carey and J.D. Pincus, An exponential formula for determining functions, Indiana Univ. Math.J. 23 (1974), 1031-1042.

[9] A. Celmins, Direkte Verfahren zur Auswertung von Schweremessungen bei zweidimensionaler Massenverteilung, Geofis. Pura Appl. 38 (1957), 81-122.

[10] D. Crowdy, J. Marshall, Constructing multiply-connected quadrature domains, SIAM J. Appl. Math. 64 (2004), 1334-1359.

[11] D. Crowdy, Quadrature domains and fluid dynamics, pp. 113-129 in [59].

[12] Ph.J. Davis, The Schwarz function and its applications, Carus Math. Mono. vol. 17, Math. Assoc. Amer., 1974.

[13] C. M. Elliott, V. Janovsky, A variational inequality approach to Hele Shaw flow with a moving boundary, Proc. Royal Soc. Edinburgh, 88A (1981), 93-107.

[14] R.J. Gardner, Geometric Tomography, Cambridge Univ. Press, 1995.

[15] G. Golub, B. Gustafsson, P. Milanfar, M. Putinar and J. Varah, Shape reconstruction from moments: theory, algorithms, and applications, Signal Processing and Image Engineering, SPIE Proceedings vol. 4116 (2000), Advanced Signal Processing, Algorithms, Architecture, and Implementations X (Franklin T. Luk, ed.), pp. 406-416.

[16] B. Gustafsson, Quadrature identities and the Schottky double, Acta Appl. Math. 1 (1983), 209-240. 
[17] B. Gustafsson, Applications of variational inequalities to a moving boundary problem for Hele-Shaw flows, SIAM J. Math. Anal. 16 (1985), 279-300.

[18] B. Gustafsson, Singular and special points on quadrature domains from an algebraic geometric point of view, J. Analyse Math. 51 (1988), 91-117.

[19] B. Gustafsson, On quadrature domains and an inverse problem in potential theory, J. Analyse Math. 55 (1990), 172-216.

[20] B. Gustafsson: A distortion theorem for quadrature domains for harmonic functions, J. Math. Anal. Appl. 202 (1996), 169-182.

[21] B. Gustafsson: Lectures on Balayage, p. 17-63 in Clifford Algebras and Potential Theory, proceedings of the summer school held in Mekrijärvi, June 24- 28, 2002. University of Joensuu Department of Mathematics, Report series no. 7, SirkkaLiisa Eriksson ed.

[22] B. Gustafsson, C. He, P. Milanfar and M. Putinar, Reconstructing planar domains from their moments, Inverse Problems 16 (2000), 1053-1070.

[23] B. Gustafsson and M. Putinar, An exponential transform and regularity of free boundaries in two dimensions, Ann. Sc. Norm. Sup. Pisa, 26 (1998), 507-543.

[24] B. Gustafsson, M. Putinar, Linear analysis of quadrature domains. II, Israel J. Math. 119 (2000), 187-216.

[25] B. Gustafsson and M. Putinar, Analytic continuation of Cauchy and exponential transforms, in S.Saitoh et al. (eds.), Analytic extension Formulas and their Applications, pp. 47-57, Kluwer Academic Publishers, Netherlands, 2001.

[26] B. Gustafsson, M. Putinar: The exponential transform: a renormalized Riesz potential at critical exponent, Indiana Univ. Math. J. 52 (2003), 527-568.

[27] B. Gustafsson, M. Putinar, H.S. Shapiro, Restriction operators, balayage and doubly orthogonal systems of analytic functions, J. Funct. Analysis 199 (2003), 332-378.

[28] B. Gustafsson, M. Sakai, Properties of some balayage operators with applications to quadrature domains and moving boundary problems, Nonlinear Anal. 22 (1994), $1221-1245$.

[29] B. Gustafsson, M. Sakai, Sharp estimates of the curvature of some free boundaries in two dimensions, Ann. Acad. Sci. Fenn. Math. 28 (2003), 123-142.

[30] B. Gustafsson, M. Sakai, On the curvature of the free boundary for the obstacle problem in two dimensions, Monatshefte für Mathematik 142 (2004), 1-5.

[31] B. Gustafsson, H.S. Shapiro, What is a quadrature domain?, pp. 1-25 in [59].

[32] B. Gustafsson, A. Vasilév, Conformal and Potential Analysis in Hele-Shaw Cells, Birkhäuser, 2006.

[33] H. Hedenmalm, N. Makarov, Quantum Hele-Shaw flow, preprint 2005. 
[34] J. W. Helton, R. Howe, Traces of commutators of integral operators, Acta Math. 135 (1975), 271-305.

[35] Yu. E. Hohlov, S. D. Howison, On the classification of solutions to the zero surface tension model for Hele-Shaw free boundary flow, Quart. Appl. Math. 54 (1994), 777-789.

[36] S. D. Howison, Cusp development in Hele-Shaw flow with a free surface, SIAM J. Appl. Math. 46 (1986), 20-26.

[37] F. John, Plane Waves and Spherical Means Applied to Partial Differential Equations, Interscince Publishers, Inc., New York, 1955.

[38] S. Karlin, W. J. Studden, Tchebycheff Systems, with Applications in Analysis and Statistics, Interscience, New York, 1966.

[39] D. Khavinson, A. Solynin and D. Vassilev, Overdetermined Boundary Value Problems, Quadrature Domains and Applications, Comput. Methods Funct. Theory 5(1) (2005), 19-48.

[40] O.S. Kouznetsova, V. Tkachev, Ullemar's formula for the Jacobian of the complex moment mapping, Complex Variables Appl. 49 (2004), 55-72.

[41] M.G. Krein and A.A. Nudelman, Markov Moment Problems and Extremal Problems, Translations of Math. Monographs, vol. 50, Amer. Math. Soc., Providence, RI, 1977.

[42] I. Krichever, A. Marshakov, A. Zabrodin, Integrable structure of the Dirichlet boundary problem in multiply conneced domains, Comm. Math. Phys. 259 (2005), 1-44. (arXiv: hep-th/0309010)

[43] I. Krichever, M. Mineev-Weinstein, P. Wiegmann, A. Zabrodin, Laplacian growth and Whitham equations of soliton theory, Physica D, 198 (2004), 1-28. (arXiv:nlin.SI/0311005)

[44] N.S. Landkof, Foundations of Modern Potential Theory, Springer-Verlag,, Berlin-Heidelberg, 1972.

[45] A. Marshakov, P. B. Wiegmann, A. Zabrodin, Integrable structure of the Dirichlet boundary problem in two dimensions, Comm. Math. Phys. 227 (2002), 131-153. (arXiv: hep-th/0109048)

[46] M. Martin and M. Putinar, Lectures on Hyponormal Operators, Birkhäuser, Basel, 1989.

[47] M. Mineev-Weinstein, P. B. Wiegmann, A. Zabrodin, Integrable structure of interface dynamics, Physical Review Letters 84 (2000), 5106-5109.

[48] M. Mineev-Weinstein, A. Zabrodin, Whitham-Toda hierarchy in the Laplacian growth problem, J. Nonlinear Math. Phys., 2001, V8, 212-218. (arXiv: hepth/0309010)

[49] F. Natterer, The Mathematics of Computerized Tomography, SIAM Classics in Applied Mathematics, Soc. Industrial and Appl. Math., Philadelphia, 2001. 
[50] P. S. Novikoff, On uniqueness for the inverse problem of potential theory, C.R. (Dokl.) Acad. Sci. URSS (N.S.) 18 (1938), 165-168.

[51] J. D. Pincus, Commutators and systems of singular integral equations. I, Acta Math. 121 (1968), 219-249.

[52] J. D. Pincus, J. Xia, D. Xia, The analytic model of a hyponormal operator with rank-one self-commutator, Integral Eq. Operator Theory 7 (1984), 516-535.

[53] J.D. Pincus, J. Rovnyak, A representation formula for determining functions, Proc. Amer. Math. Soc. 22 (1969), 498-502.

[54] M. Putinar, On a class of finitely determined planar domains, Math. Res. Lett. 1 (1994), 389-398.

[55] M. Putinar, Extremal solutions of the two-dimensional L-problem of moments, J.Funct.An. 136 (1996), 331-364.

[56] M. Putinar, Extremal solutions of the two-dimensional L-problem of moments II, J.Approx.Th. 92 (1998), 38-58.

[57] M. Putinar, On a diagonal Padé approximation in two complex variables, Numer. Math. 93 (2002), 131-152.

[58] M. Putinar, A Renormalized Riesz Potential and Applications, Adv. Constr. Approx., Vanderbilt 2003 (M.Neamtu, E. Saff, editors), 2004 Nashboro Press, Brentwood, TN, pp. 433-465.

[59] Quadrature Domains and Applications, a Harold S. Shapiro Anniversary Volume. (eds. P. Ebenfelt, B. Gustafsson, D. Khavinson, M. Putinar), Birkhäuser, 2005 .

[60] S. Richardson, Hele Shaw flows with a free boundary produced by the injection of fluid into a narrow channel, J. Fluid Mech. 56 (1972), 609-618.

[61] M. Riesz, L'intégrale de Riemann-Liouville et le problème de Cauchy, Acta Math.81 (1951), 1-223.

[62] M. Riesz, Intégrales de Riemann-Liouville et potentiles, Acta Szeged Sect. Math.9 (1937-38), 1-42.

[63] E. B. Saff, V. Totik, Logarithmic potentials with external fields, Springer Verlag, Berlin, 1997.

[64] M. Sakai, A moment problem on Jordan domains, Proc. Amer. Math. Soc. 70 (1978), 35-38.

[65] M. Sakai, Quadrature Domains, Lect. Notes Math. 934, Springer-Verlag, BerlinHeidelberg 1982.

[66] M. Sakai, Application of variational inequalities to the existence theorem on quadrature domains, Trans. Amer. Math. Soc. 276 (1983), 267-279.

[67] M. Sakai, Regularity of boundary having a Schwarz function, Acta Math. 166 (1991), 263-297. 
[68] M. Sakai, Regularity of boundaries of quadrature domains in two dimensions, SIAM J. Math. Anal. 24 (1994), 341-364.

[69] M. Sakai, Regularity of free boundaries in two dimensions, Ann. Scuola Norm Sup. Pisa Cl. Sci. (4) 20 (1993), 323-339.

[70] M. Sakai, Sharp estimates of the distance from a fixed point to the frontier of a Hele-Shaw flow, Potential Anal. 8 (1998), 277-302.

[71] M. Sakai, Linear combinations of harmonic measures and quadrature domains of signed measures with small supports, Proc. Edinburgh Math. Soc. (2) 42 (1999), 433-444.

[72] H.S. Shapiro, The Schwarz function and its generalization to higher dimensions, Uni. of Arkansas Lect. Notes Math. Vol. 9, Wiley, New York, 1992.

[73] T. Sjödin, Quadrature identities and deformation of quadrature domains, pp. 239-255 in [59].

[74] R. Teodorescu, E. Bettelheim, O. Agam, A. Zabrodin, P. Wiegmann, Normal random matrix ensemble as a growth problem, Nuclear Phys. B 704 (2005), 407444. (arXiv:hep-th/0401165)

[75] V. Tkachev, Subharmonicity of higher dimensional exponential transforms, pp. $257-277$ in [59].

[76] V. Tkachev, Ullemar's formula for the moment map. II, Linear Algebra and its Applications 404 (2005), 380-388.

[77] C. Ullemar, A uniqueness theorem for domains satisfying a quadrature identity for analytic functions, Research bulletin TRITA-MAT-1980-37, Royal Institute of Technology, 59 pp.

[78] A. N. Varchenko, P. I. Etingof, Why the Boundary of a Round Drop Becomes a Curve of Order Four, American Mathematical Society AMS University Lecture Series, Volume 3, Providence, Rhode Island 1992.

[79] P. B. Wiegmann, Aharonov-Bohm effect in the quantum Hall regime and Laplacian growth problems, Statistical field theories (Como. 2001), 337-349, NATO Sci. Ser. II Math. Phys. Chem., 73, Kluwer Acad. Publ., Dortrecht, 2002.

[80] P. B. Wiegmann, A. Zabrodin, Conformal maps and integrable structures, Comm. Math. Phys. 213 (2000), 523-538. (arXiv: hep-th/9909147)

[81] D. Xia, Spectral Theory of Hyponormal Operators, Birkhäuser, Basel, 1983.

[82] D. Xia, Hyponormal operators with finite rank self-commutator and quadrature domains, J. Math. Anal. Appl. 203(1996), 540-559.

[83] D. Yakubovich, Subnormal operators of finite type. I. Xia's model and real algebraic curves in $C^{2}$, Rev. Mat. Iberoamericana 14 (1998), 95-115.

[84] D. Yakubovich, Subnormal operators of finite type. II. Structure theorems, Rev. Mat. Iberoamericana 14 (1998), 623-681. 
[85] A. V. Zabrodin, Whitham hierarchy in growth problems, Theoretical and Mathematical Physics 142 (2005), 166-182.

[86] L. Zalcman, Some inverse problems of potential theory, Contemp. Math. 63 (1987), 337-350. 